\title{
Visualization of Triterpenoids in Mice Fed by Mass Spectrometry Imaging
}

\author{
Kyuichi Kawabata ${ }^{1}$, Yukina Tatsuta ${ }^{2}$, Shiori Nakada ${ }^{2}$, Shoma Naruse ${ }^{2}$, Shigeru Murakami ${ }^{2}$, Hajime Katano ${ }^{2}$ and \\ Shu Taira ${ }^{2 *}$ \\ ${ }^{1}$ Department of Nutrition, Kobe Gakuin University, Japan \\ ${ }^{2}$ Department of Bioscience, Fukui Prefectural University, Japan
}

Submission: August 11, 2016; Published: August 23, 2017

"Corresponding author: Shu Taira, Department of Bioscience, Fukui Prefectural University, Japan, Email: staira@fpu.ac.jp

\begin{abstract}
Two triterpenoids (ursonic acid (UNA) and oleanonic acid (ONA)) were synthesized and orally administrated to mice. After 1 and 3hours, liver, kidney and lien were took and frozen, immediately. Mass spectrometry imaging was employed to visualize UNA and ONA at a resolution of $100 \mathrm{~m}$ in the mouse liver, kidney and lien. We analyzed the localization of triterpenoids from these sections. Triterpenoids are mainly detected and localized to kidney, indicating that we imaged metabolism of target molecules.
\end{abstract}

Keywords: Functional nutrition; Imaging; Ziziphus jujube; Natural medicine

\section{Introduction}

It is well known that jujube (Ziziphus jujuba) fruit is the one of traditional herbal medicine and a nutritional food in China and South Korea. Jujube fruit contains some types of functional components, such as triterpenoic acids [1,2] flavonoids [3] and amino acids [4]. These secondary metabolites indeed contribute to change for the better of health [5] such as anti-cancer, antioxidative, and anti-obesity effects [6,7] in in vitro and in vivo. Jujube is not popular as functional foods in Japan yet, while a farm of jujube has been started from 1998 in Fukui prefecture in Japan, and its fruit is especially harvested and processed to dried fruit, extract, tea, and so on.

We have identified active triterpenoids: ursonic acids (UNA) and oleanonic acid (ONA) from methanol extract of dried jujube fruit. These compounds enhanced glucose uptake in L6 myotubes cell in a GLUT4-dependent fashion [8]. Hence, there is a possibility that the triterpenoids modulate in vivo blood glucose level via the upregulation of muscle glucose uptake. On the other hand, bioavailability and biokinetics of triterpenoids have not been fully investigated. Commonly, ADME of small active compounds including triterpenoids were analyzed by high-performance liquid chromatography (HPLC) method [9]. Therefore, there are no studies that the direct imaging of compounds with pharmacological activity such as the Ziziphus jujube after ingestion intake.
In order to facilitate increased direct visualization of biomolecules during biological analysis, mass spectrometry is used to distinguish individual molecules from each other. Mass spectrometric imaging (MSI) enables simultaneous detection of multiple analytes even in the absence of the targetspecific markers such as antibodies [10]. MSI commonly was used in medical research to reveal the mechanism of diseases $[11,12]$. In this study, the ONA and UNA as triterpenoids were synthesized and identified by MS. After oral administration of these semi-synthesized triterpenoids to mouse, its liver, kidney and lien were visualized at a resolution of $100 \mu \mathrm{m}$ using the matrix assisted laser desorption/ionization (MALDI) time-offlight (TOF) MALDI-TOF-MSI technique. Our study is the first to report on the visualization of localization of triterpenoids for disposition using MSI-TOF-MSI technique.

\section{Materials and Methods}

\section{Semi-synthesis of oleanonic acid (ONA) and ursonic acid (UNA)}

Oleanolic acid and ursonic acid were subjected to Jones oxidation [13] (chromium trioxide/sulfuric acid in acetone) to respectively obtain ONA and UNA with yields over 95\%. 


\section{Mouse model of ONA and UNA administration}

Seven-week-old female C57BL/6NJcl mice (20-22g; Clea Japan, Tokyo, Japan) were used in accordance with the institutional Animal Experimental Guidelines of Fukui Prefectural University. They were fed laboratory chow and allowed free access to water. For the single administration experiment, we used a feeding needle to administer ONA or UNA $(10 \mathrm{mg}$ ) containing normal saline solution to mice, respectively. Mice fed a normal saline solution were used as the control.

\section{Preparation of frozen cross-sections of mice organ}

All mice $(n=6)$ were anesthetized with $2 \%$ isoflurane by anesthetic instrument (SN-487-OT, Natsume SEISAKUSHO Co. Ltd, Japan). Blood was corrected from cervical vein and immediately added heparin (1unit/mL). Corrected blood was centrifuged ( $5 \mathrm{~min}, 3,000 \mathrm{rpm}, 4{ }^{\circ} \mathrm{C}$ ) to obtain plasma solution. The liver, kidney, and lien were dissected by a surgical scissors and tweezers at room temperature, then achieved flash-freezing in liquid $\mathrm{N}_{2}$ and stored at $-80{ }^{\circ} \mathrm{C}$ until use. The organs were embedded in $2 \% \mathrm{CMC}$ and cut into serial sections $(8 \mu \mathrm{m})$ using a cryostat (CM-3050 S; LEICA, Germany) at $-25{ }^{\circ} \mathrm{C}$ and thawmounted on indium tin oxide (ITO)-coated slides for MSI.

\section{Determination of triterpenoids profile in the plasma by high performance liquid chromatography (HPLC)}

The plasma sample $(100 \mu \mathrm{L})$ of control or triterpenoidstreated was added to $100 \mu \mathrm{L}$ of ethyl acetate and mixed, respectively. The organic layer was recovered and dried to correct triterpenoids material. The dried residue was added to $100 \mu \mathrm{L}$ of ethyl alcohol. The extraction from the liver was analyzed by HPLC. HPLC analysis-ODS column (COSMOCORE,
2.6C18, 2.1ID $\times 100 \mathrm{~mm}$, Nakarai Tesque Inc, Kyoto, Japan) was used. Flow, $0.4 \mathrm{~mL} / \mathrm{min}$. isocratic elution, $0.1 \%$ formic acid (FA)/ water (A) and acetonitrile/0.1\% FA (B); 0-25min, 80\% B, 25$30 \mathrm{~min}, 100 \% \mathrm{~B}$; for wash. The injection volume was $1 \mu$ l. target molecules were detected by $208 \mathrm{~nm}$ wavelengths

\section{MALDI-MSI of triterpenoids localization in mice organ}

Target MS plates were coated with a $10 \mathrm{mg} \mathrm{ml}^{-1}$ solution of $\alpha$-cyano-4-hydroxycinnamic acid (CHCA, Nakarai Tesque Inc, Kyoto, Japan) matrix. Semi-synthesized triterpenoids, ONA and UNA which dissolved in ethyl acetate (Wako Pure Chemicals, Japan) as standards, was dropped on a coated plate. For confirmation of ionization of standard triterpenoids, we used MALDI-TOF instrument (Ultraflex extreme, Bruker Daltonik $\mathrm{GmbH}$ ) using yttrium aluminium garnet (YAG laser) emissions at $355 \mathrm{~nm}$. Saturated CHCA was dispersed in methanol and was centrifuged, and the supernatant fluid was sprayed on a glass slides with the sections using an airbrush (nozzle caliber, $0.2 \mathrm{~mm}$ ). The section surface was irradiated with 100 laser shots in the negative ion detection mode of the mass spectrometer. To detect the laser spot area, sections were scanned and laser spot areas $(1,000$ shots $)$ were detected with a spot-to-spot center distance of $100 \mu \mathrm{m}$ in each direction of the liver, kidney and lien, respectively.

\section{Results and Discussion}

We confirmed deprotonated signal at $\mathrm{m} / \mathrm{z} 453.3$ was detected corresponding to ULA (m/z 455.4) to UNA, and OLA (m/z 455.4) to ONA, respectively (Figure 1 ). These mass difference indicated oxidation of oleanolic acid or ursolic acid by Jones method.

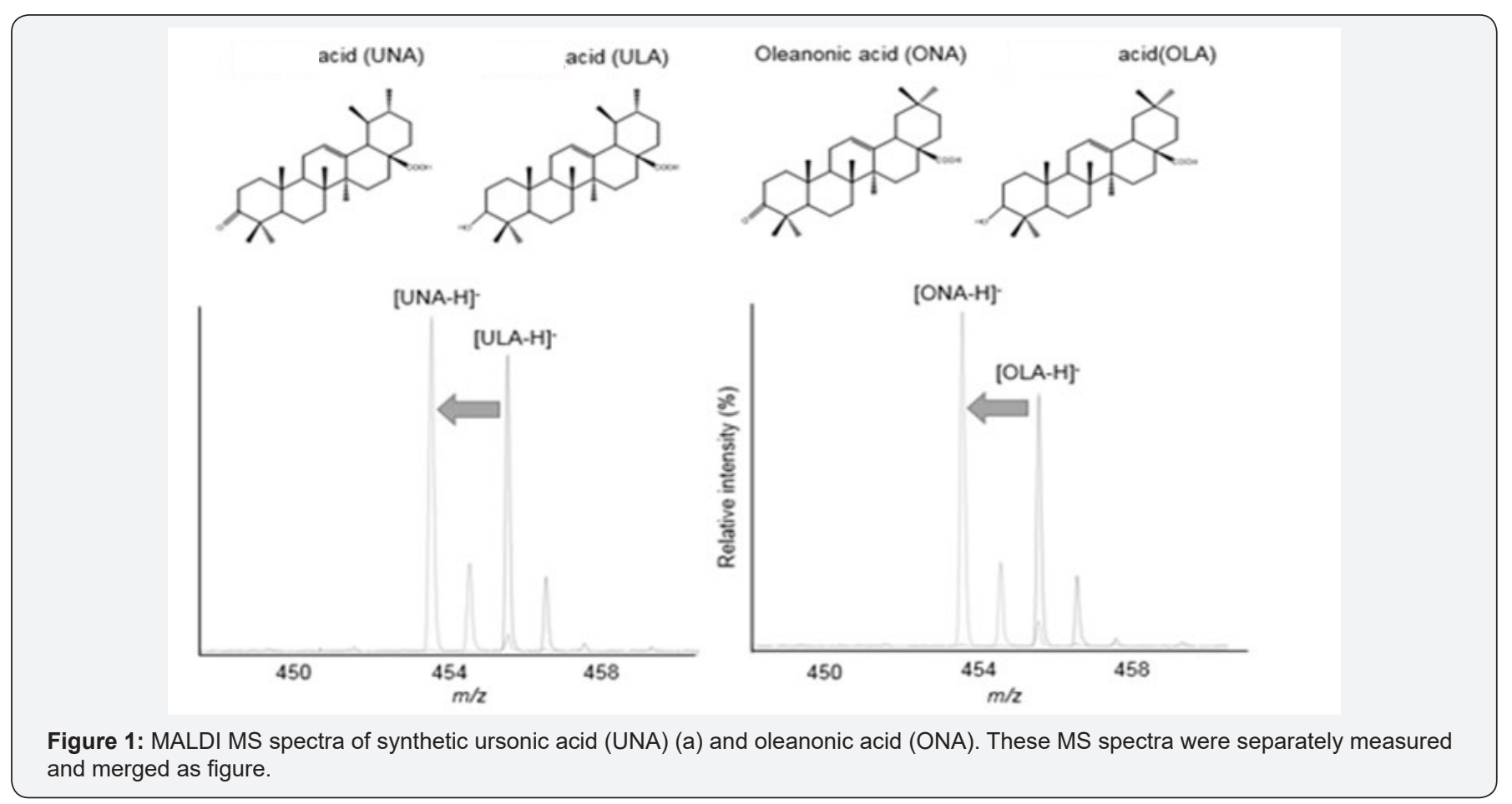


In this time, UNA and ONA were separately administrated to mice due to same molecular weight. The result of HPLC analysis for plasma showed that the ration of peak areas of the control, 1 and 3 hours were 1:3:4 for UNA and 1:2:2 for ONA. Both detectedUNA and -ONA from these triterpenoids-treated mice increased in blood and did not show the concentration difference on time lag, suggesting that both triterpenoids were gradually absorbed into body at chorionic tissue of small intestine.
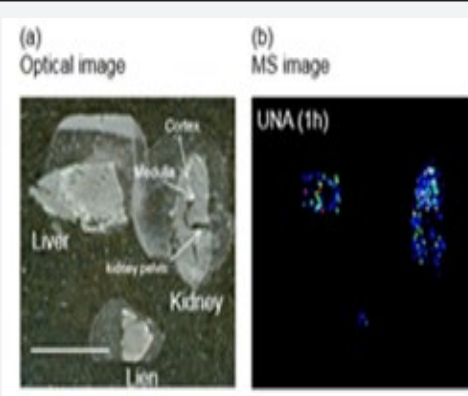

\section{Merged image}
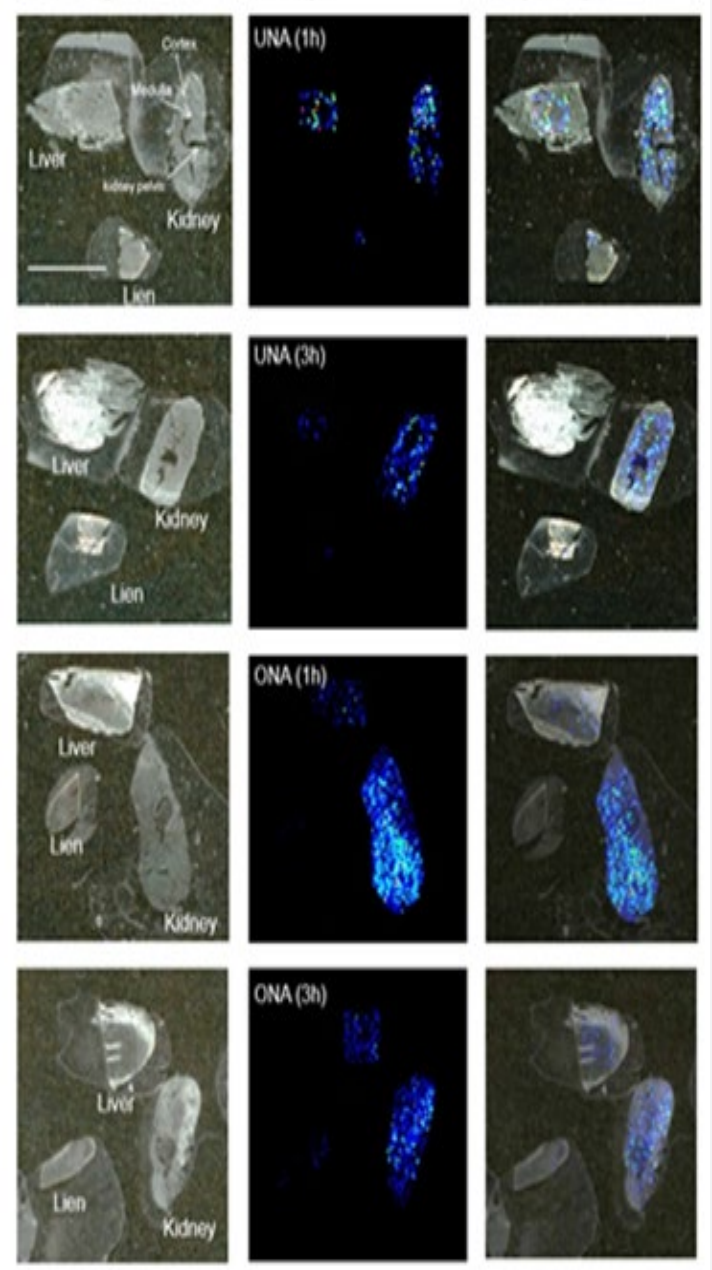

Figure 2: MALDI-mass spectrometric imaging (MSI) of UNA and ONA. Optical images of mouse liver, kidney and lien sections (a) and MS image for UNA and ONA (b) and merged image (c). MS spectra reconstructed as ion images for $\mathrm{m} / \mathrm{z}$ 453.3. Total MS intensity at the sections were measured to quantify the MS intensity. Obtained values were commuted to per unit area. $n=$ 3. The scale bar is $5 \mathrm{~mm}$.

Next, we analyzed existence and localization of UNA and ONA, visually by MSI method (Figure 2). A number of highintensity signals, including UNA or ONA, were detected in the mass spectrum obtained from the organs seeped with CHCA. For the liver, both UNA and ONA were marginally detected. In a similar way, from lien section, we did not confirm the signals correlated with these triterpenoids. On the other hand, both UNA and ONA could be clearly imaged on kidney. One hour passed after ingestion of UNA or ONA, both UNA and ONA localized at outer and inner region where is may cortex and medulla of kidney. Whereas, three hours passed after ingestion of UNA or ONA, these triterpenoids mainly localized medulla of kidney. Semi- quantitative analysis was performed by matching the MS intensities of kidney for UNA. The ratio of signal intensity of the control, 1 and 3 hours was 1:1.4:1, respectively. For that of ONA, the ratio of signal intensity of the control and 1 and 3 hours was $1: 2: 1$. The ratio intensity of ONA was larger than that of UNA, may suggesting that excretion of ONA was faster than that of UNA. The result of plasma analysis showed that of UNA was higher than that of ONA at $1 \mathrm{~h}$, indicating that ONA was rapidly distributed to organ and excreted. The different plasma levels of the triterpenoids as mentioned above may support this speculation. These results indicated that these triterpenoids were normally adsorbed in body and through the kidney and egested as urine at kidney region. We could detect UNA and ONA as intact compound not those of metabolite due to a high concentration of administrated triterpenoids in this experimental condition. Thus, in this study, we focused on original compounds, while these triterpenoids may be subjected to the detoxification metabolism in liver, such as hydroxylation, sulfonation and glucuronidation. The detection and analysis will be addressed in near future.

\section{Conclusion}

MALDI-MS is an effective technique for the identification and visualization of triterpenoids. UNA and ONA delivered to liver and kidney although these triterpenoids were excreted out by metabolism system. ONA preferably excreted out at short period compared with UNA. The difference between UNA and ONA for structure was just position of a methyl group. In the future, we consider the correlation between the structure of triterpenoids and egestion period using other triterpenoids such as betulonic acid and betulinic acid and intend to obtain an effective spatial resolution image by nanoparticle-assisted laser desorption/ ionization MS imaging to reveal a more accurate distribution of molecules such as their cellular resolution.

\section{Acknowledgment}

This research was supported by a KAKENHI (JP16K04890 to S.T.) from the Japan Society for the Promotion of Science, a Lotte grant and Cosmetology foundation grant to S.T., and in part by a grant from the Hokuriku Industrial Advancement Center to K.K. This work was partly supported by the Nanotechnology Platform Program of the Ministry of Education, Culture, Sports, Science and Technology (MEXT), Japan.

\section{References}

1. Guo S, Duan J, Qian D, Tang Y, Wu D, et al. (2015) Content variations of triterpenic acid, nucleoside, nucleobase, and sugar in jujube (Ziziphus jujuba) fruit during ripening. Food Chem 167: 468-474.

2. Alqahtani A, Hamid K, Kam A, Wong KW, Abdelhak Z, et al. (2013) The Pentacyclic Triterpenoids in Herbal Medicines and Their Pharmacological Activities in Diabetes and Diabetic Complications. Curr Med Chem 20(7): 908-931. 
3. Chen J, Li Z, Maiwulanjiang M, Zhang WL, Zhan JYX, et al. (2013) Chemical and Biological Assessment of Ziziphus jujuba Fruits from China: Different Geographical Sources and Developmental Stages. J Agric Food Chem 61(30): 7315-7324.

4. Guo S, Duan JA, Qian D, Tang Y, Qian Y, et al. (2013) Rapid Determination of Amino Acids in Fruits of Ziziphus jujubaby Hydrophilic Interaction Ultra-High-Performance Liquid Chromatography Coupled with TripleQuadrupole Mass Spectrometry. J. Agric Food Chem 61(11): 27092719.

5. Gao QH, Wu CS, Wang M (2013) The Jujube (Ziziphus Jujuba Mill.) Fruit: A Review of Current Knowledge of Fruit Composition and Health Benefits. J Agric Food Chem 61: 3351-3363.

6. Plastina P, Bonofiglio D, Vizza D, Fazio A, Rovito D, et al. (2012) Identification of bioactive constituents of Ziziphus jujube fruit extracts exerting antiproliferative and apoptotic effects in human breast cancer cells. J Ethnopharmacol 140(2): 325-332.

7. Yu L, Jiang BP, Luo D, Shen XC, Guo S, Duan JA, Tang YP (2012) Bioactive components in the fruits of Ziziphus jujuba Mill. against the inflammatory irritant action of Euphorbia plants. Phytomedicine 19(34): 239-244.

8. Kawabata K, Kitamura K, Irie K, Naruse S, Matsuura T, et al. (2017) Triterpenoids isolated from Ziziphus jujuba enhance glucose uptake activity in skeletal muscle cells Increased muscle glucose uptake by jujube triterpenoids. J Nutri Sci Vitaminol 63(3): 193-199.

9. Guo S, Duan JA, Tang YP, Yang YN, Qian DW, et al. (2010) Characterization of Triterpenic Acids in Fruits of Ziziphus Species by HPLC-ELSD-MS. J Agric Food Chem 58(10): 6285-6289.

10. Stoeckli M, Chaurand P, Hallahan DE, Caprioli RM (2001) Imaging mass spectrometry: a new technology for the analysis of protein expression in mammalian tissues. Nat Med 7(4): 493-496.

11. Sugiura Y, Taguchi R, Setou M (2011) Visualization of spatiotemporal energy dynamics of hippocampal neurons by mass spectrometry during a kainate-induced seizure. PLoS One 6(3): e17952.

12. Taira S, Yamaguchi N, Morimoto S, Tatsuta Y, Katano H, et al. (2016) Liver disease risk of xenobiotics due to percutaneous absorption revealed by Nano-PALDI imaging mass spectrometry. International Journal of Recent Scientific Research 7: 12589-12592.

13. Bowden K, Heilbron IM, Jones ERH, Weedon BCL (1946) Researches on acetylenic compounds. Part I. The preparation of acetylenic ketones by oxidation of acetylenic carbinols and glycols. Journal of the Chemical Society (Resumed): 39-45.

\begin{tabular}{l} 
Your next submission with Juniper Publishers \\
will reach you the below assets \\
- Quality Editorial service \\
- Swift Peer Review \\
- Reprints availability \\
- E-prints Service \\
- Manuscript Podcast for convenient understanding \\
- Global attainment for your research \\
- Manuscript accessibility in different formats \\
( Pdf, E-pub, Full Text, Audio) \\
- Unceasing customer service \\
Track the below URL for one-step submission \\
https://juniperpublishers.com/online-submission.php \\
\hline
\end{tabular}

